
allemande

\title{
Vers un renouveau de la répression pénale des violences autour des manifestations sportives : une préoccupation transnationale
}

Chronique juridique

\section{Sandie Calme}

\section{OpenEdition}

Journals

Édition électronique

URL : https://journals.openedition.org/allemagne/617

DOI : 10.4000/allemagne.617

ISSN : 2605-7913

\section{Éditeur}

Société d'études allemandes

Édition imprimée

Date de publication : 29 décembre 2017

Pagination : 505-510

ISSN : 0035-0974

\section{Référence électronique}

Sandie Calme, «Vers un renouveau de la répression pénale des violences autour des manifestations sportives : une préoccupation transnationale », Revue d'Allemagne et des pays de langue allemande [En ligne], 49-2 | 2017, mis en ligne le 29 décembre 2017, consulté le 28 mai 2021. URL : http:// journals.openedition.org/allemagne/617 ; DOI : https://doi.org/10.4000/allemagne.617 


\section{Chronique juridique}

\section{Vers un renouveau de la répression pénale des violences autour des manifestations sportives: une préoccupation transnationale}

\section{- Sandie Calme*}

Les violences récurrentes aux abords des stades de football lors de spectacles sportifs appellent une réponse pénale particulière. Concrètement, la jurisprudence pénale est confrontée à des cas particuliers où les limites constitutionnelles de la liberté d'expression semblent méconnues, comme cela est exprimé de façon exemplaire au travers de la jurisprudence du Tribunal constitutionnel fédéral allemand. La réponse répressive connaît également, de façon différenciée, une appréhension à caractère constitutionnaliste de la part des juridictions françaises. Au fond, une perspective humanitariste semble se dessiner à l'échelle transnationale.

L'arrêt du Tribunal constitutionnel fédéral allemand en date du 16 janvier 2017 (1 BvR 1593/16) apporte de nouveau un éclairage essentiel sur une problématique pénale d'actualité.

En l'espèce, le requérant avait fait l'objet d'une condamnation pénale pour injure sur la base de l'article $\$ 185$ du Code pénal allemand (Strafgesetzbuch, StGB). Il avait procédé, par correspondance, à l'acquisition d'un écusson portant l'abréviation anglophone injurieuse A.C.A.B. et de deux autres, symboliques, portant les nombres 13 et 12 , et les avait accolés à son gilet. C'est avec ce gilet qu'il se présenta, en mars 2015, à un spectacle de football. Lors du contrôle d'entrée, la police étant présente afin d'assurer la sécurité de l'événement, l'un des policiers voyant les écussons fit procéder au contrôle collectif du visiteur, d'où la prise d'acte de cette attitude considérée comme une injure. Il en résulta pour l'intéressé une condamnation pénale par le Tribunal d'instance (Amtsgericht) au versement d'une amende de 80 euros sur quatre-vingt-dix jours. En

* Docteur en droit, LL.M. (Francfort-sur-le-Main, Allemagne), avocate au barreau de Paris. 
appel, la peine fut simplement transformée en cinquante jours-amende de 60 euros. Pour le Landgericht, juridiction d'appel, le plaignant s'était rendu coupable de l'infraction pénale d'injure en portant des inscriptions dénigrant les autorités policières par abréviation et codage chiffré. Il avait manifesté cette expression par le port du gilet avec un écrit visible envers les personnes lésées, au nombre de quatre policiers. Ceuxci, en tant que communauté de personnes, avaient été affectés par une désignation collective. L'expression humiliante s'était adressée aux fonctionnaires de police qui avaient pris part, en cette qualité, au contrôle. Ainsi, leur groupe se distinguait de toute généralité et se composait d'un nombre d'agents déterminé. La juridiction avait acquis la conviction que le plaignant avait porté le gilet en connaissance de la signification des écrits et qu'il était conscient de la fatalité de rencontrer des policiers en venant assister au spectacle sportif, policiers qui étaient chargés du contrôle de sécurité, et que, de cette manière, les policiers seraient évidemment au contact de cette expression. La juridiction avait bien pris en considération qu'une expression adressée à des membres d'un groupe en général ne se rapportait pas à un groupe de personnes déterminable en elle-même, en ce qu'un tel groupe fait partie d'un ensemble fongible de personnes. Toutefois, elle notait que l'appelant s'était placé par exprès dans une situation dans laquelle il devait très certainement rencontrer quelques fonctionnaires de police. On ne pouvait pas exiger qu'il connaisse nommément les policiers en question qu'il aspirait à injurier comme préalable pour le punir.

L'Oberlandesgericht, juridiction de degré supérieur, rejetait le pourvoi en cassation du plaignant sur la base de la prise de position de l'avocat général, selon laquelle on était en présence d'un ralliement personnalisé à certains policiers, dans la mesure où le plaignant devait forcément, en se rendant au spectacle, fatalement rencontrer un grand nombre de fonctionnaires de police concrètement identifiables.

C'est sur la base de la liberté fondamentale constitutionnelle de l'article 5 alinéa $1^{\text {er }}$, première phrase, de la Loi fondamentale, à savoir le droit de libre expression, que l'intéressé présentait un recours en contrôle de constitutionnalité.

Ce recours a prospéré, il a été déclaré recevable afin de voir reconnaître les droits fondamentaux du plaignant, le Tribunal constitutionnel fédéral ayant justement eu l'occasion de trancher la problématique auparavant. Il en allait de l'impact du droit fondamental de liberté d'expression dans l'interprétation et dans l'application des normes pénales circonscrites par ce droit fondamental.

La violation de l'article 5 alinéa $1^{\text {er }}$, première phrase, de la Loi fondamentale, était ainsi reconnue.

Pour le Tribunal constitutionnel, la condamnation pénale en cause lèse la liberté d'expression du requérant car le port du gilet avec les inscriptions litigieuses entre dans le champ de protection du droit fondamental. En effet, les opinions, contrairement aux affirmations factuelles, se caractérisent par la subjectivité et sont dignes de protection, qu'elles soient fondées ou non, émotionnelles ou rationnelles, avec ou sans valeur, dangereuses ou innocentes aux yeux de quiconque.

La liberté d'expression connaît les limites des lois générales, des dispositions légales de protection de la jeunesse et du droit à la dignité des personnes, l'article $₫ 185$ du Code pénal allemand étant une loi générale propre à contenir la liberté d'expression. 
Toutefois, l'intrusion de la présente condamnation pénale dans la liberté d'expression n'est pas justifiée parce que les exigences constitutionnelles quant à l'application et à l'interprétation de l'article $\$ 185$ du Code pénal comme tempérament à la liberté d'expression ne sont pas garanties.

Il s'agit de garantir la teneur de la liberté d'expression afin de ne pas la vider de son sens et de tout contenu.

Un ensemble de personnes somme toute très général, fongible et de facto indéterminé ne saurait être considéré comme étant l'objet d'une infraction pénale d'injure en l'espèce.

Il manque des éléments établissant que le plaignant se soit placé en connaissance de cause à la portée des forces de l'ordre de la police afin de les confronter à son expression écrite.

Pour le Tribunal constitutionnel fédéral, si la décision attaquée repose sur cette erreur, il n'est pas exclu que la juridiction de renvoi, juge du fond, en vienne à une autre décision par une conceptualisation différenciée.

Cet arrêt était l'occasion pour le Tribunal de réitérer une appréciation du même ordre portée à une situation analogue en rapport avec ces mêmes expressions écrites à l'occasion de spectacles sportifs (deux arrêts du 17 mai 2016, 1 BvR257/14 et 1 BvR 2150/14).

La condamnation initiale se basait sur l'article $\$ 185$ du Code pénal allemand (Strafgesetzbuch), selon lequel l'injure est punie d'un an d'emprisonnement ou d'une amende, voire de deux ans d'emprisonnement ou d'une amende si elle est commise au moyen d'une violence. Cette disposition se trouvait confrontée avec l'article $\$ 90$ alinéa $1^{\text {er }}$ de la loi du Tribunal constitutionnel fédéral (Bundesverfassungsgerichtsgesetz), qui autorise quiconque à saisir ce tribunal s'il estime être lésé dans un de ses droits fondamentaux ou quant aux droits mentionnés au sein de certains articles de la Loi fondamentale. En l'occurrence, c'est l'article 5 relatif à la liberté d'expression qui fonde ce recours.

Ici, le Tribunal constitutionnel fédéral se base sur sa jurisprudence sur la libre expression dans le cadre des oppositions politiques (BVerfG·Beschluß vom 7. Dezember 1976 Az. 1 BvR 460/72; voir également 13 avril 1994, BVerfGE 90, 241 (241)) et également relative au dénigrement de la profession de soldat (10 octobre 1995, BVerfGE 93, 266).

Cette conceptualisation de la liberté d'expression en la matière n'est pas sans rappeler une jurisprudence très attendue du droit français. Selon l'article L332-16-1 du Code des sports, créé par la loi n² 2011-267 du 14 mars 2011,

«Le ministre de l'Intérieur peut, par arrêté, interdire le déplacement individuel ou collectif de personnes se prévalant de la qualité de supporter d'une équipe ou se comportant comme tel sur les lieux d'une manifestation sportive et dont la présence est susceptible d'occasionner des troubles graves pour l'ordre public.

L’arrêté énonce la durée, limitée dans le temps, de la mesure, les circonstances précises de fait qui la motivent ainsi que les communes de point de départ et de destination auxquelles elle s'applique.

Le fait pour les personnes concernées de ne pas se conformer à l'arrêté pris en application des deux premiers alinéas est puni de six mois d'emprisonnement et d'une amende de $30000 €$. 
Dans le cas prévu à l'alinéa précédent, le prononcé de la peine complémentaire d'interdiction judiciaire de stade prévue à l'article L. 332-11 pour une durée d'un an est obligatoire, sauf décision contraire spécialement motivée.»

Dans un arrêt du Conseil d'État en date du 22 janvier 2016 (nº 396307, inédit au recueil Lebon), l'Association nationale des supporters (ANS) demandait au juge des référés du tribunal administratif de Nantes de suspendre l'exécution d'un arrêté du 15 janvier 2016 par lequel un préfet avait interdit à tout supporter d'un club de football de vendre ou d'acheter des billets permettant d'assister à un match de championnat contre un autre club, et avait interdit aux personnes se prévalant de la qualité de supporter de ce club ou se comportant comme telles ainsi qu'à toute personne ayant appartenu à une association dissoute de ce club d'accéder, de circuler ou de stationner sur un certain périmètre pendant une certaine période. L'association exprimait le souhait qu'il soit ordonné au préfet de procéder au réexamen de sa décision. Par une ordonnance du 21 janvier 2015, le juge des référés du tribunal administratif de Nantes avait rejeté ces demandes. Par une requête ultérieure auprès du juge des référés du Conseil d'État, l'association demandait d'annuler cette ordonnance, de faire droit à sa demande de première instance et de mettre à la charge de l'État une certaine somme au titre de l'article L. 761-1 du code de justice administrative.

L'association prétendait que le juge des référés avait entaché son ordonnance de contradiction de motifs et d'erreur de droit quant à la portée de son contrôle, que la condition d'urgence était remplie, que l'arrêté litigieux portait une atteinte grave et manifestement illégale à la liberté d'aller et venir, à la liberté d'association, à la liberté de réunion et à la liberté d'expression, que l'arrêté contesté est manifestement illégal, le préfet de la Loire-Atlantique ne justifiant, d'après elle, ni de la nécessité ni de la proportionnalité des mesures d'interdiction. L'arrêté ayant été pris tardivement, elle estimait qu'il méconnaissait les dispositions de la convention européenne sur la violence et les débordements de spectateurs lors des manifestations sportives, méconnaissant également le principe d'égalité et l'exigence de clarté et d'intelligibilité de la norme.

Sur la base de la convention européenne sur la violence et les débordements de spectateurs lors de manifestations sportives, du code du sport et du code de justice administrative, le Conseil d'État concluait « qu'il appartient aux autorités de l'État d'assurer la préservation de l'ordre public et sa conciliation avec les libertés fondamentales que sont notamment la liberté d'aller et venir, la liberté d'association, la liberté de réunion et la liberté d'expression " pour reconnaître la rectitude de l'arrêté. En effet, il résultait de l'instruction que des incidents violents étaient survenus à plusieurs reprises, en marge de rencontres opposant les deux équipes. Dans ces conditions, et compte tenu de l'inimitié particulière qui régnait entre les supporters de ces deux clubs, il ne résultait pas manifestement de l'instruction que les interdictions posées par l'arrêté litigieux ne seraient pas nécessaires à la préservation de l'ordre public. Il ne résultait pas davantage manifestement de l'instruction que, dans les circonstances de l'espèce et eu égard au contexte caractérisé par la forte mobilisation des forces de l'ordre pour lutter contre la menace terroriste et parer au péril imminent ayant conduit à la déclaration de l'état d'urgence, ainsi que pour assurer la sécurité publique lors de la manifestation des opposants au projet d'aéroport du Grand Ouest devant se tenir concomitamment à proximité, des mesures moins contraignantes que celles édictées par l'arrêté litigieux 
seraient de nature à éviter la survenance des troubles graves à l'ordre public qu'elles ont pour but de prévenir. L'arrêté ne portait pas d'atteinte grave et manifestement illégale aux libertés fondamentales invoquées par l'association nationale des supporters. La requête était rejetée.

Dans un arrêt ultérieur du Conseil d'État en date du 10 août 2016, sur le même fondement juridique, associé à la loi n 2016-987 du 21 juillet 2016 prorogeant l'application de la loi n ${ }^{\circ} 55-385$ du 3 avril 1955 relative à l'état d'urgence et portant mesures de renforcement de la lutte antiterroriste, le Conseil d'État reprenait la même analyse relative à la liberté d'expression ( $\mathrm{n}^{\circ} 402256$, inédit au recueil Lebon).

La requête de l'Association de défense et d'assistance juridique des intérêts des supporters demandait au juge des référés du Conseil d'État de suspendre l'exécution d'un arrêté du 5 août 2016 par lequel le ministre de l'Intérieur avait interdit le déplacement des supporters d'un club de football lors d'une rencontre avec un autre club. Elle souhaitait qu'il soit enjoint au ministre de l'Intérieur de réétudier la situation pour trouver une solution intermédiaire, notamment par le recours au dialogue, et qu'il soit mis à la charge de l'État une certaine somme au titre de l'article L. 761-1 du code de justice administrative. Elle estimait que la condition d'urgence était remplie en raison de l'imminence de la rencontre sportive, qu'il était porté une atteinte grave et manifestement illégale à la liberté d'aller et venir, d'association, de réunion et d'expression de l'association ainsi qu'à celle de ses membres. Pour elle, l'arrêté méconnaissait les stipulations de la convention européenne sur la violence et les débordements de spectateurs lors de manifestations sportives et notamment de matches de football du 19 août 1985 ainsi que la convention sur une approche intégrée de la sécurité, de la sûreté et des services lors des matches de football et autres manifestations sportives du 3 juillet 2016, car le ministre n'avait pas engagé de dialogue préalable pour organiser en lien avec l'association un déplacement encadré et régulé des supporters. Elle lui reprochait une erreur d'appréciation, faute de caractériser les risques de troubles graves à l'ordre public ni les circonstances de temps et de lieu justifiant l'interdiction de déplacement. De son point de vue, le ministre de l'Intérieur avait édicté une mesure manifestement disproportionnée et l'arrêté contesté était entaché d’illégalité en raison de la tardiveté de sa publication.

Il résultait de l'instruction que les matches de football entre les deux équipes avaient donné lieu, à plusieurs reprises et également récemment, de la part de certains groupes de supporters, à des troubles graves à l'ordre public, y compris lors d'une rencontre ayant fait l'objet d'une interdiction identique. Ainsi, la rencontre en cause était un événement à haut risque. La présence du club de supporters concerné devait nécessairement impliquer une augmentation substantielle des forces de police localement présentes. Il n'apparaissait pas que puissent être prises immédiatement des mesures moins contraignantes de nature à éviter les troubles graves à l'ordre public susceptibles de se produire. Dans ces circonstances particulières de temps et de lieu et en dépit de la sévérité des restrictions imposées aux supporters du club visé, les mesures prises par l'arrêté ministériel contesté ne caractérisaient pas une atteinte manifestement illégale aux libertés fondamentales en cause à savoir la liberté d'aller et venir, la liberté d'association, la liberté de réunion et la liberté d'expression. D’où le rejet de la requête. 
Ce contexte révèle une réelle préoccupation interétatique. La Convention européenne sur la violence et les débordements de spectateurs lors de manifestations sportives et notamment de matches de football (Strasbourg, 19.VIII.1985), citée par la jurisprudence française, est un texte émanant des démarches humanitaristes du Conseil de l'Europe. Il engage ses États Parties à prendre, dans son domaine, des mesures coordonnées ainsi que des initiatives de coopération internationale. Il a été dénoncé par la France le 17 mars 2017 et n'est jamais entré en vigueur en Allemagne. S'y substitue la Convention du Conseil de l'Europe sur une approche intégrée de la sécurité, de la sûreté et des services lors des matches de football et autres manifestations sportives (Saint-Denis, 3.VII.2016). Ratifiée par la France le 6 février 2017 et par Monaco le 2 mars 2017, elle doit entrer en vigueur après trois ratifications. 